\title{
Reduction of EMC Emissions in Mixed Signal Integrated Circuits with Embedded LIN Driver
}

\author{
Pavel HARTL, Marian KUBAN, Pavel HORSKY \\ ON Design Czech, Videnska 125, 61900 Brno, Czech Republic \\ Pavel.Hartl@onsemi.com, Marian.Kuban@onsemi.com, Pavel.Horsky@onsemi.com \\ Manuscript received April 06, 2016
}

\begin{abstract}
This paper describes several methods for reduction of electromagnetic emissions (EME) of mixed signal integrated circuits (IC). The focus is on the impact that a LIN bus communication block has on a complex IC which contains analog blocks, noisy digital block, microcore $(\mu C)$ and several types of memories. It is used in an automotive environment, where EMC emission reduction is one of the key success factors. Several proposed methods for EME reduction are described and implemented on three test chips. These methods include current consumption reduction, internal on-chip decoupling, ground separation and different linear voltage regulator topologies. Measurement results of several fabricated test chips are shown and discussed.
\end{abstract}

\section{Keywords}

EMC, emissions, ground splitting, linear voltage regulator, automotive industry, LIN bus driver

\section{Introduction}

The automotive industry is known for a harsh environment for electronic circuits, mainly with respect to used semiconductors, starting with a very wide temperature operating range, ESD pulses, transients present on supply and signal lines, requirements for very low emission and high immunity to electromagnetic disturbances [1].

Due to continuous progress in manufacturing technology of integrated circuits (IC), the electromagnetic compatibility (EMC) of electronic systems plays increasing role in circuit performance. The number of devices inside a single integrated circuit and the clock speed increase resulting in higher electromagnetic emissions [2]. Integrated circuits for automotive applications have to be designed with a strong focus on reduction of the EMC emissions.

This paper starts in Sec. 2 with a short introduction to EMC emissions of integrated circuits. The primary focus of the paper is on reduction of EMC emissions of a complex automotive mixed signal IC containing substantial analog content including output drivers, ADCs and DACs, and a digital core including memories and LIN bus drivers. The device is described in Sec. 3. In Sec. 4, several methods for electro-magnetic emissions (EME) reduction are discussed and demonstrated on a test chip where the impact of different grounding schemes and supply regulator topologies were tested. Measurement results are presented, compared with expectations and conclusions are drawn.

\section{EMC Emissions of ICs}

\subsection{Emission Sources}

Main on chip EMC emission sources include [2], [3]:

- Clock-driven blocks, synchronized logic

- Memory read/write/refresh

- IO switching

- Switching On/Off heavy loads

- Oscillators

- Switching of fast comparators

- Floating domains with high $\mathrm{dV} / \mathrm{dt}$ transients

The major EMC emission source of the analyzed IC was the digital section. The digital block is the origin of EMC emissions due to the transient current flowing through each elementary logic gate. Supply current peaks occur whenever the logic gate changes its output state either at rising (from logic level " 0 " to " 1 ") or falling edge (from "1" to "0"), see Fig. 1 [4]. The combination of several thousands of gates leads to significant current peaks.

All on-chip output drivers controlling IC pins are important for EMC emissions as well. Special attention has
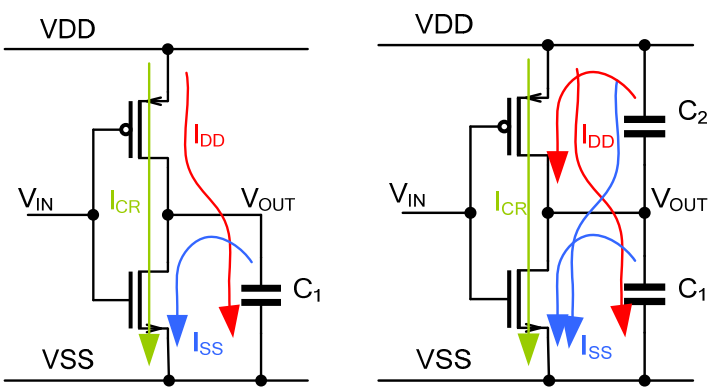

Fig. 1. Current path in simple CMOS inverter [4]. 
to be paid to the pins driving wires leaving a module to a cabling harness. High parasitic capacitances and long wires can easily cause significant emissions.

\subsection{Coupling Paths}

The emissions generated on a chip can be coupled to the external environment by way of three different principles [5]:

- Conducted emission through the supply lines caused by switching activity of the core and buffers, which induces current drops on the supply pins of the chip.

- Coupling by the IOs: The current spikes provoked by the internal core may be measured on the IOs of the circuit. The PCB wires connected to the IO serve as antennas for the energy.

- On-die radiated coupling: The internal currents inside the chip are responsible for the radiated emissions.

The following chapters are focused on the reduction of conducted emissions in the supply and ground lines and also on the reduction of coupling by IOs. The on-die radiated emissions are not considered in this case since the operating clock frequency of the evaluated chip and its corresponding wave length is negligible considering the die dimensions.

\subsection{Emission Reduction}

Examples of good design recommendations include separating signal and power routing, shielding of the sensitive signal tracks, dedicated ground and supply lines for noisy and sensitive blocks [5], [6], EME controlled performance of noisy circuits (i.e. controlled transitions of the power drivers, spreading of the operating frequency) [7], local on-chip/off-chip supply decoupling [8], [9], appropriate package selection [10], die to package bonding strategy and last, but not least, careful selection of the device layout floor-plan [5].

\subsection{Emission Measurement Methods}

EMC standards used in the automotive industry for evaluation of EMC emissions include CISPR25 [11].

The measurement test setup used for the EMC emission performance evaluation compliant with CISP25 with an artificial network (AN) is shown in Fig. 2.

\section{Original Design}

The goal of this work was to reduce EMC emissions of a complex mixed signal IC which was not passing EMC emission tests. The original chip was designed in $\mathrm{ON}$ Semiconductor high voltage smart power $350 \mathrm{~nm}$ technology. The block diagram of the initial device depicted in

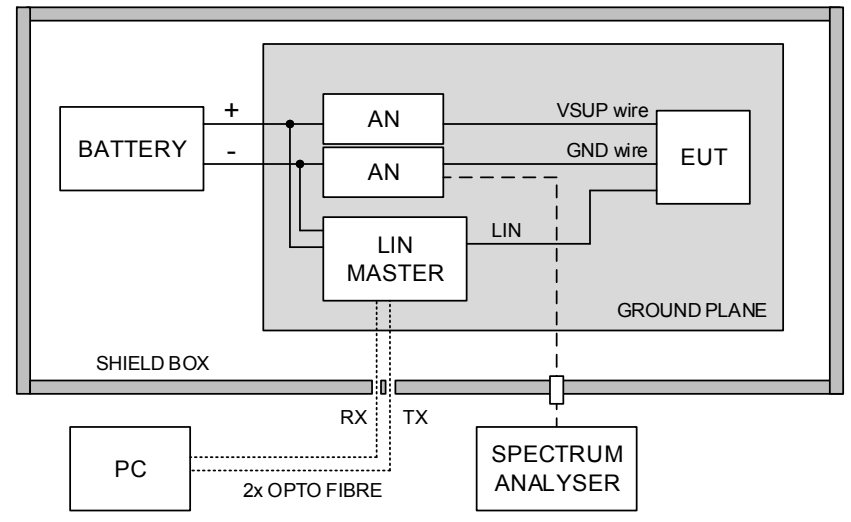

Fig. 2. EMC conducted emission measurement setup according to the CISPR25 (AN) with power line remotely grounded [11].

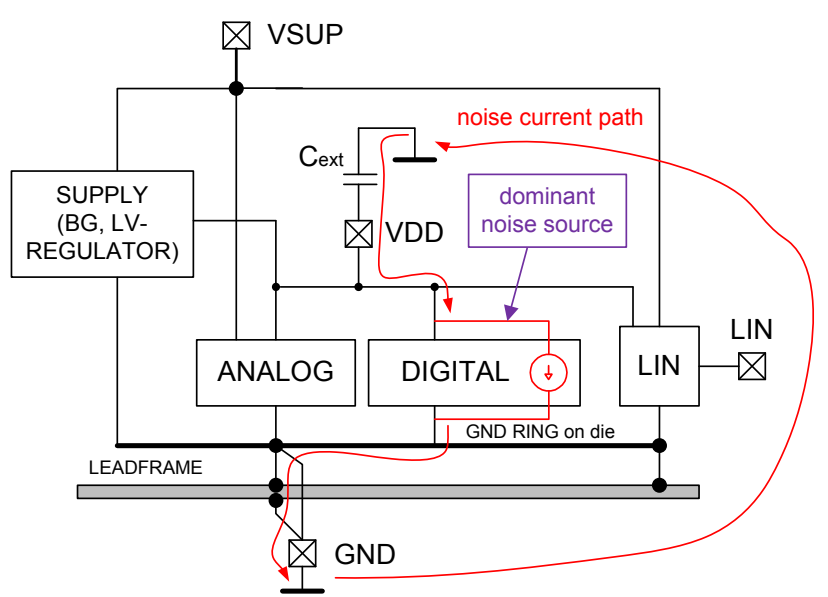

Fig. 3. The original device block diagram and noise current path.

Fig. 3 contains a low-voltage supply (VDD) regulator block which supplies a variety of analog circuitry (like output drivers, measurement channels with $\mathrm{ADC}$, current sources, DAC, etc.), digital circuitry (custom logic, $\mu \mathrm{C}$, memories) and a LIN block. The module containing this IC is connected to the other modules via a wiring harness with VSUP, LIN and GND wire.

The dominant noise source in the IC is the digital block with synchronous logic running at a main clock frequency of approximately $8 \mathrm{MHz}$. The digital peak current is delivered from the external decoupling capacitor $\mathrm{C}_{\mathrm{ext}}$ connected between the VDD pin and PCB ground. The peak current flow is depicted in the block diagram in Fig. 3. Part of the high frequency current can be delivered from the VSUP pin, depending on the high frequency transfer function of the integrated linear voltage regulator. The VSUP pin is also decoupled by an external capacitor, which will take part of the high frequency current, but the residual part of the current can still flow through the supply line and cause significant EMC emissions, simply because of the length of the current path loop.

Measured EMC emissions on the GND line according to the CISPR25 method are shown in Fig. 4. Significant peaks above target limit are visible in the frequency range 


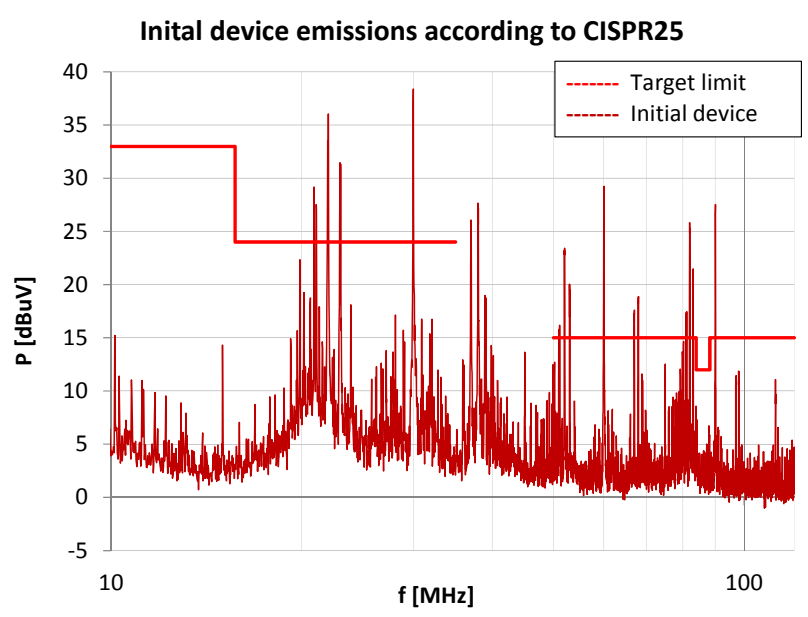

Fig. 4. The original device EMC emissions according to the CISPR25, AN method.

from $10 \mathrm{MHz}$ up to the $120 \mathrm{MHz}$. The main peaks are at frequencies $30 \mathrm{MHz}$ and $60 \mathrm{MHz}$ (multiples of the main oscillator frequency).

\section{Reduction of EMC Emissions}

After a detailed study of the EMC emission sources and coupling paths, three different test chips were designed, manufactured and measured for EMC emissions. Different packages were used to split supply and ground connections. In addition, the connection of a lead frame to the PCB ground has been done in a different way. The original device had the lead frame connected to the ground in a single point, primarily to increase the ESD robustness, leading to the shared grounding of the sensitive and noisy blocks. All of the test chips had the lead frame connected at two points in order to eliminate this shared ground path of the noisy and sensitive blocks (required ESD robustness was also reached in this configuration).

\subsection{Splitting of Grounds and Local Decoupling}

The following three techniques for EMC emissions reduction were applied in the test chips:

- Reduction of the current consumption of the noise source,

- Peak current decoupling as close as possible to the noise generator to minimize the length of the noise current loop,

- Splitting of the supply and ground lines of the analog blocks, noisy digital block and IOs.

For the current consumption reduction of the complete digital section, each sub block was analyzed with respect to the overall current consumption. The standard digital gate library was changed to be more efficient with respect to a relatively low main clock frequency. The result is a smaller unit transistor size, resulting in much smaller input capacitance of each digital gate and a smaller peak current consumption. Also the current consumption of the embedded memories was checked with respect to the clock speed and access time. total average current consumption of the digital block was The successfully decreased by $1 / 3$ while maintaining the main clock frequency at $8 \mathrm{MHz}$.

Figure 3 shows the peak current decoupling path which is too long and badly designed. The following actions were done in order to shorten this path. To make the path as short as possible, any free space in the digital section was filled by MOSFET-type capacitors connected between the digital ground and the low-voltage supply (VDD). In addition, all power routing and signal routing channels in the test chips were filled by MOSFET-type capacitors. The internal decoupling is very effective for EMC emission reduction because the intrinsic capacitance is placed very close to the noise sources and the parasitic resistances and capacitances of the connections are minimized. The total accumulated on-chip decoupling capacitance was roughly $5 \mathrm{nF}$.

Because the internal capacitance between the ground and the low voltage supply was large enough for proper device functionality (under EMC), the external decoupling capacitor on low voltage supply $\mathrm{C}_{\mathrm{ext}}$ was not needed [12-14]. As a result the peak current path is maintained only locally on the chip.

The power routing of the noisy blocks was separated from the sensitive analog blocks and the noisy LIN block, see Fig. 5. The LIN block was connected over the LIN bus to the external environment and acts as an antenna for the EMC emissions. The ground connection of the LIN block was separated from the main ground connection. Ground shifts caused by the digital or IO driver switching were no longer emitted by the LIN bus.

The EMC emissions of the test chip 1 were measured and compared with the performance of the original device (see Fig. 6).

The measured emissions of the test chip 1 dropped about 10 to $20 \mathrm{~dB}$ and the peaks were able to fit within the

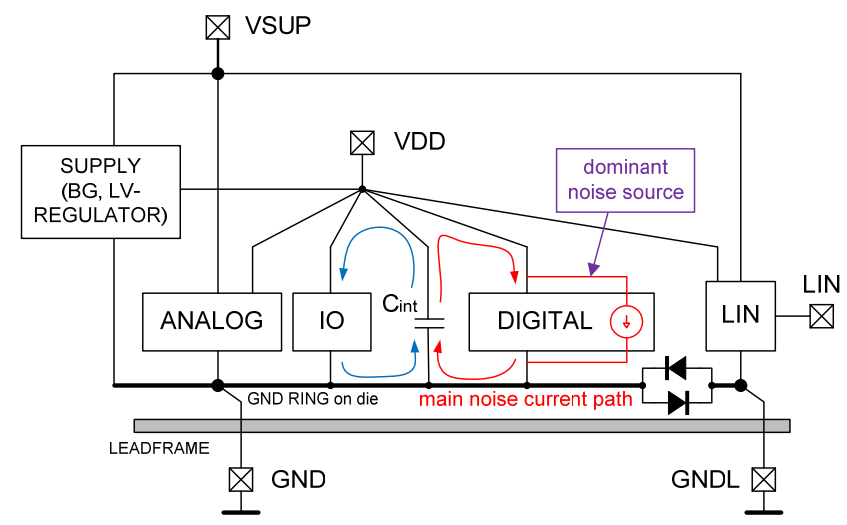

Fig. 5. The test chip version 1, block diagram and noise current path. 


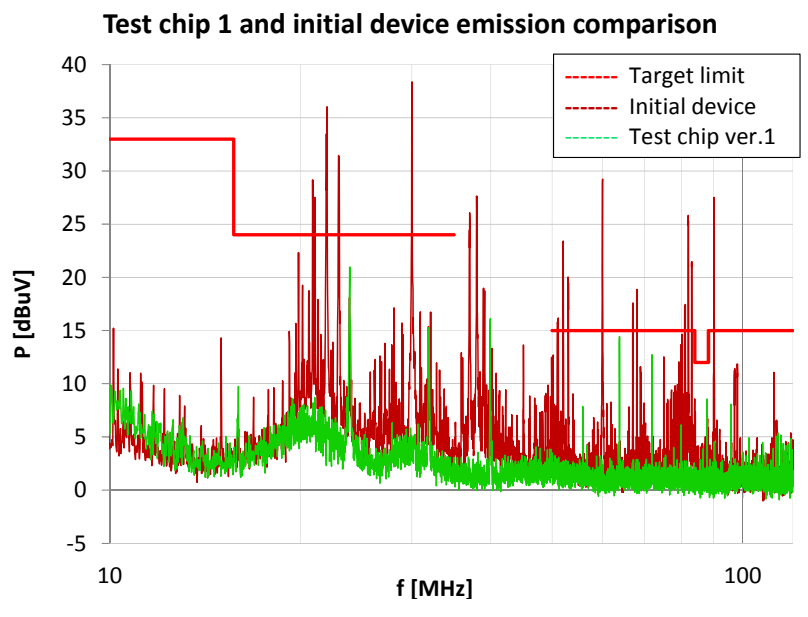

Fig. 6. EMC emission comparison of test chip with the original device, CISPR25, AN method.

target limits. The biggest reduction was seen at the fourth harmonic frequency of the main oscillator around $32 \mathrm{MHz}$ (oscillator of the original device was not calibrated so the main clock frequency was shifted to $7.5 \mathrm{MHz}$ ).

\subsection{Splitting of Ground Pins}

The original device and the test chip 1 were assembled in the SOIC16 package. Due to its limited pin count, the number of pins dedicated to ground and supply connections were limited. To overcome this limitation a QFN 32 package was used and the ground and supply connections were extended in the test chip 2 (see Fig. 7).

The ground lines were split. For instance, the IO drivers have separate ground connections and some ground pins and the bond wires were placed in parallel in order to decrease the impedance of the connection to the PCB and, consequently, the voltage drop. The EMC emission performance comparison between the two package types is shown in Fig. 8.

The measurement results of test chips with different packages show that the emissions can be further decreased at some frequencies by 3 to $10 \mathrm{~dB}$ simply by a proper separation of the ground and supply lines.

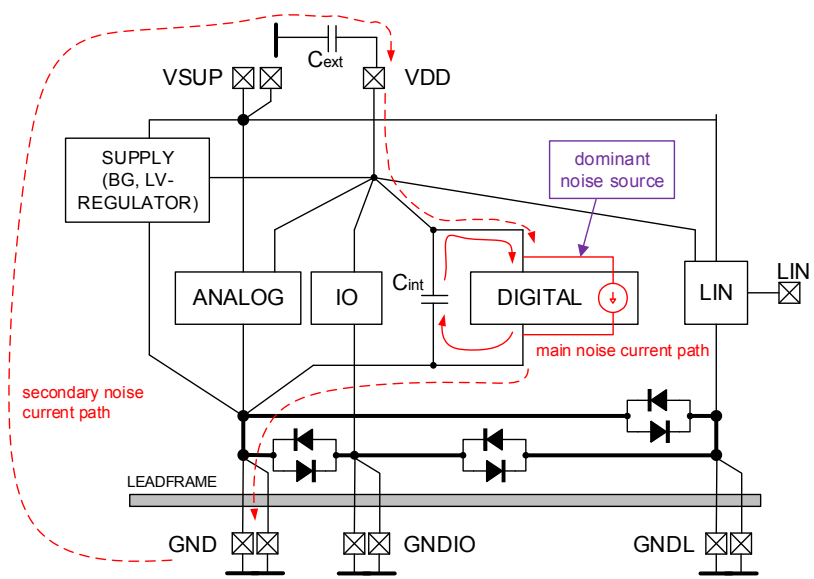

Fig. 7. Block diagram and noise current paths of test chip \#2 and \#3.

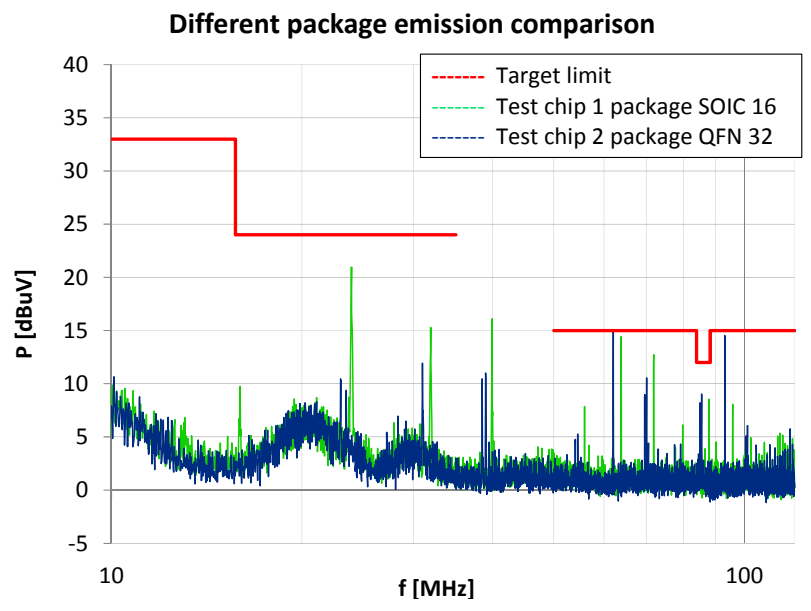

Fig. 8. EMC emission comparison of test chip in different package options, CISPR25, AN method.

\subsection{Linear Voltage Regulator Topologies}

The impact of the linear voltage regulator topology on EMC emissions was tested using two types of regulators. The original device and test chip 1 and 2 were manufactured with source follower linear voltage regulators with an NMOS-type output transistor (Fig. 9). The dominant pole resides inside the regulator block so there is no need of an external stabilization capacitor. The decoupling capacitor for ripple reduction can be kept internally, on chip.

Test chip 3 was equipped with an LDO type regulator with a PMOS-type output transistor, which was stabilized by a dominant pole on an external decoupling capacitor. All internal poles in the regulator were well above the gain bandwidth of the regulator. An external stabilization capacitor $\mathrm{C}_{\mathrm{EXT}}$ of $1 \mu \mathrm{F}$ was connected between VDD and GND pins.

An EMC comparison of the regulators comprised of PMOS and NMOS-type output stages has been further discussed in [15]. The voltage regulator with a PMOS output stage has been found superior compared to the regulator with an NMOS output stage [15]. Results of the comparative measurement of the conducted EMC emissions are shown in Fig. 10. Test chips in QFN32 packages were measured.
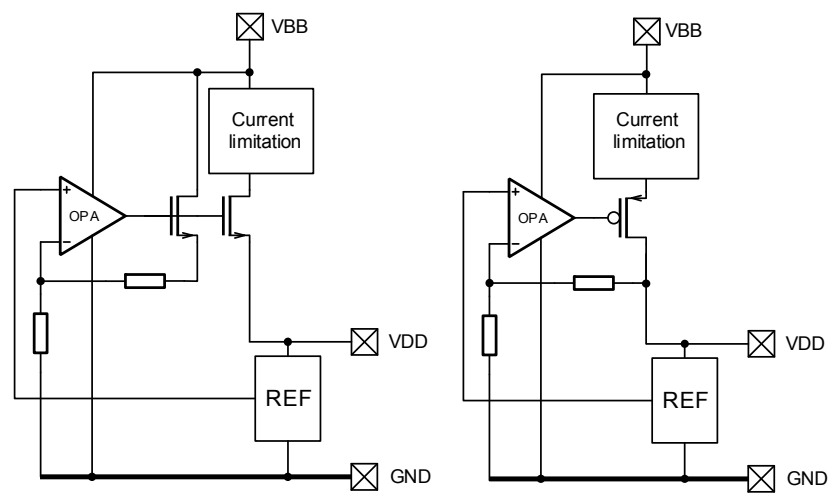

Fig. 9. Linear voltage regulator topologies, source follower (left) and LDO (right). 


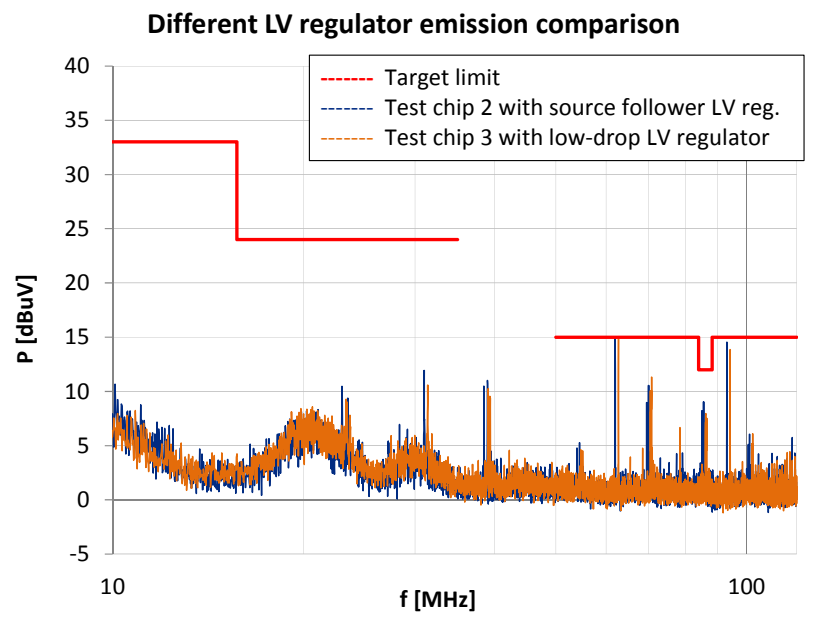

Fig. 10. EMC emission comparison of different low voltage regulator structures used in test chips.

No significant difference in EMC emissions between the two regulator topologies was observed. This could be the result of the effectiveness of all previously disclosed methods for EMC emission reduction where a minimum of emissions is coupled through the regulators and the remaining coupling paths are outside of the regulators.

\section{Figure of the Test Chip}

An overview of the layout placement of the main blocks of the fabricated test chip is shown in Fig. 11. The majority of the area is occupied by the digital section, followed by the IO and LIN bus blocks.

\section{Conclusions}

This paper focuses on practical EMC emissions improvements to a complex mixed signal IC for automotive applications with an embedded LIN driver in the frequency range from $10 \mathrm{MHz}$ to $120 \mathrm{MHz}$. Several techniques were discussed and implemented in the test chips and measured. Measurement results were compared with the initial device. Significant improvements of about 10 to $20 \mathrm{~dB}$ were

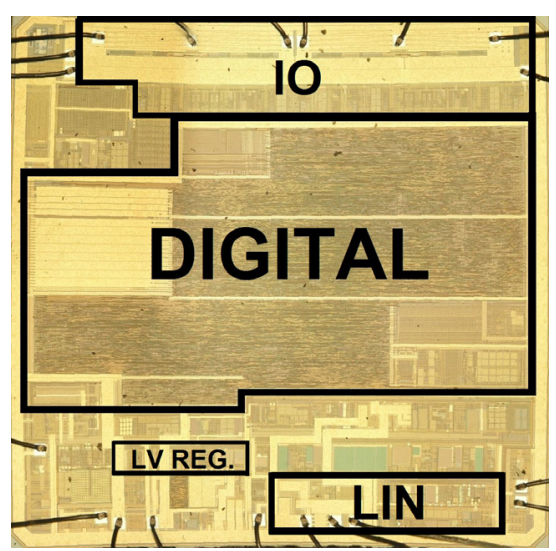

Fig. 11. The fabricated test chip photo. achieved by current consumption reduction, internal on chip decoupling and separation of the LIN block ground. Further reduction of the EMC emissions in a lower frequency range was reached with the QFN32 package where more pins were available and the supply and ground pins were further split. Finally, the two different topologies of linear voltage regulators were compared and no significant difference in EMC performance of the complete test chips was observed.

\section{References}

[1] LAUDE, D. IC design considerations for the harsh automotive environment. In Proceedings of the IEEE Custom Integrated Circuits Conference. San Diego (CA, USA), 1994, p.319-326. DOI: 10.1109/CICC.1994.379710

[2] SICARD, E., BENDHIA, S., RAMDANI, M. Electromagnetic Compatibility for Integrated Circuits. Springer, 2006. ISBN: 9780387266015.

[3] FIORI, F.L. Reducing SoC electromagnetic emissions by design. In 15th IEEE International Conference on Electronic, Circuits and Systems. 2008, p. 422-425. DOI: 10.1109/ICECS.2008.4674880

[4] HORSKY, P. DRE1 - Design of Analog ICs. Brno (Czech Rep.): Brno University of Technology, 2008. Textbook, $128 \mathrm{p}$.

[5] BEN DHIA, S., RAMDANI, M., SICARD, E. Electromagnetic Compatibility of Integrated Circuits: Techniques for Low Emission and Susceptibility. Berlin: Springer, 2006, 473 p. ISBN: 0-38726600-3.

[6] FIORI, F., MERLIN, M. A new grounding scheme to reduce the electromagnetic emission of smart-power system-on-chip. IEEE Transactions on Power Electronics, 2012, vol. 27, p. 224-243. DOI: 10.1109/TPEL.2010.2068312

[7] DEUTSCHMANN, B., ILLIING, R., AUER, B. Edge shaping to reduce the electromagnetic emissions. In Proceedings of 10th International Symposium on Electromagnetic Compatibility. York (UK), 2011. p. 742-745. ISBN: 978-1-4577-1709-3.

[8] JOGHOON KIM, HYUNGSOO KIM, WOONGHWAN RYU, et al. Effects of on-chip and off-chip decoupling capacitors on electromagnetic radiated emission. In 48th IEEE Electronic Components and Technology Conference. Seattle (USA), 1998, vol. 2, p. 610-614. DOI:10.1109/ECTC.1998.678758

[9] FUJI, H., KOBAYASHI, Y., SUDO, T. Evaluation of power supply noise reduction by implementing on-chip capacitance. In 8th Workshop on Electromagnetic Compatibility of Integrated Circuits (EMC Compo). Dubrovnik (Croatia), 2011, p. 219-223. ISBN: 978-1-4577-0862-6.

[10] MERLIN, M., FIORI, F. Impact of package parasitics on the EMC performance of smart power SoCs. In IEEE European Microelectronics and Packaging Conference (EMPC 2009). Rimini (Italy), 2009, p. 1-6. ISBN: 978-1-4244-4722-0.

[11] CISPR 25: Limits and Methods of Measurement of Radio Disturbance Characteristics for the Protection of Receivers used on Board Vehicles. 2nd ed. 2002

[12] RINCON-MORA, G. Analog IC Design with Low-Dropout Regulators. New York: McGraw-Hill, 2009, 372 p. ISBN: 9780071608930 .

[13] MILLIKEN, R. J., SILVA-MARTINEZ, J., SANCHEZ-SINEN$\mathrm{CIO}$, E. Full on-chip CMOS low-dropout voltage regulator. IEEE 
Transactions on Circuits and Systems, 2007, vol. 54, no. 9, p. 1879-1890. DOI: $10.1109 /$ TCSI.2007.902615

[14] CHAVA, C. K., SILVA-MARTINEZ, J. A frequency compensation scheme for LDO voltage regulators. IEEE Transactions on Circuits and Systems, 2004, vol. 51, no. 6, p. 1041-1050. DOI: 10.1109/TCSI.2004.829239

[15] LAVERY, K., SMITH, R. Impact of linear regulator topology on integrated circuit emissions. In IEEE International Symposium on Electromagnetic Compatibility. Honolulu (HI, USA), 2007, vol. 2, p. 1-4. DOI: 10.1109/ISEMC.2007.82

\section{About the Authors ...}

Pavel HARTL was born in Boskovice. He received his M.Sc. degree in Electronics and Communication from the Department of Radio Electronics, Brno University of Technology, Czech Republic in 2004. He is currently a student in the graduate Electronics and communication techniques program of the same department. He is a member of a design group at ON Design Czech, ON Semiconductor company. His interests include a mixed signal IC design for automotive products with focus on stepper motor drivers and EMC.

Marian KUBAN was born in Martin. He received his B.Sc. and M.Sc. degree in Electronics and Communication from the Department of Radio Electronics, Brno University of Technology, Czech Republic in 2010 and 2013, respectively. He is currently a student in the graduate Electronics and communication techniques (D-EST) program of the same department. He is a member of an analog ASIC design group at ON Design Czech, ON Semiconductor company. His interests are analog electrical circuits, signal processing and programming.

Pavel HORSKY received the M.Sc. degree in 1994 in Radio Electronics and the Ph.D. degree in 1998 in the field of metrology at the Brno University of Technology, Czech Republic. In 2011 he became an associated professor at the same university. Currently he is leading an analog ASIC design group at ON Design Czech, ON Semiconductor company. His professional interests include an analog/ mixed signal IC design for automotive products with focus on EMC, ESD and reliability. 